Research Article

\title{
Study on Behind Helmet Blunt Trauma Caused by High-Speed Bullet
}

\author{
Zhihua Cai $\mathbb{D}^{1},{ }^{1}$ Xingyuan Huang $\mathbb{D}^{1},{ }^{1}$ Yun Xia, ${ }^{1}$ Guibing Li $\mathbb{D}^{1},{ }^{1}$ and Zhuangqing Fan $\mathbb{D}^{2}$ \\ ${ }^{1}$ College of Mechanical and Electrical Engineering, Hunan University of Science and Technology, Xiangtan, China \\ ${ }^{2}$ Daping Hospital, Army Medical University, Chongqing, China \\ Correspondence should be addressed to Zhuangqing Fan; fanzhuangqing@163.com
}

Received 22 May 2019; Revised 28 October 2019; Accepted 26 November 2019; Published 19 February 2020

Academic Editor: Mohammad Rahimi-Gorji

Copyright (C) 2020 Zhihua Cai et al. This is an open access article distributed under the Creative Commons Attribution License, which permits unrestricted use, distribution, and reproduction in any medium, provided the original work is properly cited.

\begin{abstract}
The mechanism of Behind Helmet Blunt Trauma (BHBT) caused by a high-speed bullet is difficult to understand. At present, there is still a lack of corresponding parameters and test methods to evaluate this damage effectively. The purpose of the current study is therefore to investigate the response of the human skull and brain tissue under the loading of a bullet impacting a bullet-proof helmet, with the effects of impact direction, impact speed, and impactor structure being considered. A human brain finite element model which can accurately reconstruct the anatomical structures of the scalp, skull, brain tissue, etc., and can realistically reflect the biomechanical response of the brain under high impact speed was employed in this study. The responses of Back Face Deformation (BFD), brain displacement, skull stress, and dura mater pressure were extracted from simulations as the parameters reflecting BHBT risk, and the relationships between BHBT and bullet-proof equipment structure and performance were also investigated. The simulation results show that the frontal impact of the skull produces the largest amount of $\mathrm{BFD}$, and when the impact directions are from the side, the skull stress is about twice higher than other directions. As the impact velocity increases, BFD, brain displacement, skull stress, and dura mater pressure increase. The brain damage caused by different structural bullet bodies is different under the condition of the same kinetic energy. The skull stress caused by the handgun bullet is the largest. The findings indicate that when a bullet impacts on the bullet-proof helmet, it has a higher probability of causing brain displacement and intracranial high pressure. The research results can provide a reference value for helmet optimization design and antielasticity evaluation and provide the theoretical basis for protection and rescue.
\end{abstract}

\section{Introduction}

Behind Helmet Blunt Trauma (BHBT) means that when a high-energy bullet and explosive debris impact bullet-proof helmets, the helmet is deformed without penetration and the back of the helmet is exposed to brain force or shock waves transmitted to the brain which cause damage to the brain $[1,2]$. Rafaels et al. analyzed more than 6000 cases of bullets and fragments in war, antiterrorism, and peacekeeping and found that more than $70 \%$ of injured patients were wearing bullet-proof helmets and other individual protective equipment, of which more than $50 \%$ suffered brain injuries [3]. However, the mechanism of BHBT is still not clear. Therefore, understanding the injury mechanism of BHBT and strengthening the protection of the brain are the urgent problems to be solved.
For the study of nonpenetrating injuries in individual equipment, because it is not possible to experiment with living people, cadaveric head experiments, animal experiments, dummy experiments, and digital simulation methods are the main approaches. The cadaver skull is the most similar substitute for living organisms; it can be employed for studies of the biomechanical response and injury mechanism of tissues effectively. However, the sources of fresh and complete cadaver specimens are very limited, the acquisition of sample is very difficult and costly, and there is poor repeatability. The animal experimental model can simulate the response of biological tissues under impact to a certain extent, but there are some differences between the animal and the human body in anatomical structure and tissue response. The finite element method can replace the biomechanical test to a certain extent and study the damage of various parts of the skull and brain. 
In the previous ten years, Jazi et al. [4], Yang and Dai [5], Pintar et al. [6], and Tse et al. [7] established the human head biomechanical simulation model and used cadaveric experiments for validations; the finite element method was used widely from then on.

In recent years, a large number of scholars have established head finite element models through various methods and approaches. Through the efforts of generations and generations, finite element models with higher precision have been developed on the original simple model, for example, the ULP [8] (University of Louis Pasteur Model) in France, the WSUBIM [9] (Wayne State University Brain Injury Model) in the United States, the KTH [10] (Kungliga Tekniska Hogskolan) in Sweden, the UCDBTM [11] (University College Dublin Brain Trauma) in Ireland, and the GHBMC [12] (Global Human Body Models Consortium). What is more, the NRL-Simpleware head model [13] is already being used for exploring head injury in the military by the U.S. Naval Research Laboratory.

At present, many scholars have conducted research on the parameters of helmet protection. Pintar et al. [6], Rodríguez-Millán et al. [14], and Palta et al. [15], using the combination of experiment and simulation, studied the injury mechanism of helmet protection under the impact of a bullet. Li et al. [16] established and validated the clayinjected human brain simulation model. Tham et al. [17] used light gas guns to conduct the experiments and simulations. Hai et al. [18] used the landrace and the physical model to study the brain injury caused by different bullet speeds. Rafaels et al. [3] carried out the cadaver experiment of the brain injury caused by the bullet impacting a bullet-proof helmet; Palomar et al. [19] used the finite element method to study the injury of the skull caused by different bullet speeds. What is more, there are also studies on other parameters. For example, Yang and Dai [5] established a human brain biomechanical simulation model and carried out simulation analysis of brain damage caused by the bullet impact at different angles and different positions. Tan et al. [20] researched on both experiments and numerical simulations of frontal and lateral ballistic impacts on the Hybrid III headform equipped with Advanced Combat Helmets $(\mathrm{ACH})$. The results show that a finite element model of human brain biomechanics with high fidelity can accurately simulate the biomechanical response and damage of the brain under the impact of a bullet. Jazi et al. [4] established a human brain simulation model to study the biomechanical response of the brain when the bullet impacts the bulletproof helmet. The parameters of different brain cushions, different impact angles, and different impact positions on the brain were analyzed. Aare and Kleiven [21] used the brain finite element model to simulate the biomechanical response of the brain under the parameters of helmet stiffness and different impact angles.

The purpose of current study is to investigate the response of the human skull and brain tissue under the loading of a bullet impacting the helmet, with the effects of impact direction, speed, and impactor structure being considered. To achieve this, a human brain finite element model which can accurately reconstruct the anatomical structures of the scalp, skull, brain tissue, etc., and can realistically reflect the biomechanical response of the bullet-proof-protected brain under high impact speed was used in this study.

The responses of BFD, brain displacement, skull stress, and dura mater pressure were extracted from simulations as parameters to reflect $\mathrm{BHBT}$ risk; the relationships between BHBT and bullet-proof equipment structure and performance were also investigated. The findings can provide a better understanding on the biomechanical response of the brain under the loading of a bullet impacting a bullet-proof helmet.

\section{Materials and Methods}

2.1. Establishment of Finite Element Models. The helmet uses the established and verified US army advanced combat helmet [22] which is shown in Figure 1(a). The helmet model used for this study has 7 foams. The foam models are shown in Figure 1(b). The size of the cylindrical foam is $150 \times 30 \mathrm{~mm}$ in diameter and height. The length, width, and height of the rectangular blocks are $90 * 55 * 30 \mathrm{~mm}$ and $90 * 85 * 30 \mathrm{~mm}$; the element size is $3 \mathrm{~mm}$. The advanced combat helmet liner is a polyurethane foam material; the gasket material is a hard foam with stress-strain behavior with load rate sensitivity. According to the properties of the rigid foam material, the MAT_LOW_DENSITY_FOAM (MAT_57) is used in the LS-DYNA material library. The material density is $6.1 \times 10^{-8} \mathrm{~kg} / \mathrm{mm}^{3}$, the Young's modulus is $8.4 \mathrm{MPa}$, and the relationship between stress and strain was taken from a uniaxial tensile test, and the stress-strain curve which is shown in Figure 1(c) is fixed by MATLAB.

The handgun, fragmentation, and rifle bullets are all constructed by the software PROE (Parametric Technology Corporation, Massachusetts, United States) to create a geometric model. The eight-node hexahedral element is used for meshing in the finite element preprocessing software HyperMesh (Altair Engineering Inc., Troy, MI, USA). The element size of the handgun bullet is $2 \mathrm{~mm}$ [20]. The whole model contains 21616 nodes and 1695 elements, and the mass is $8 \mathrm{~g}$. The finite element model of fragmentation [23] is using AISI 4340 steel. The whole model contains 14283 nodes and 12584 elements with a mass of $1.13 \mathrm{~g}$. The rifle bullet finite element model [24] is using a $7.62 \mathrm{~mm}$ rifle projectile whose warhead is divided into three parts: armor, steel core, and lead core. The whole model contains 8640 nodes and 5389 elements with a mass of $10.98 \mathrm{~g}$. The finite element model diagrams are shown in Figure $1(\mathrm{~d})$. The bullet material parameters are shown in Table 1.

The established finite element model of the brain $[25,26]$ is improved, the skin and the skull have surface-to-surface contact, and other brain tissues are using a self-contact algorithm. The cerebrospinal fluid was modified to be an ALE mesh during the preprocessing, the material was changed to $*$ MAT_ELADTIC_PLUID (MAT_1), and the unit algorithm was ELFORM $=12$. The keyword $* \mathrm{CON}$ STRAINED_LAGRANGE_IN_SOLID is set to achieve the coupling between the structure and the fluid, so that the "skull-cerebrospinal fluid-brain" has a fluid-solid coupling relationship. 


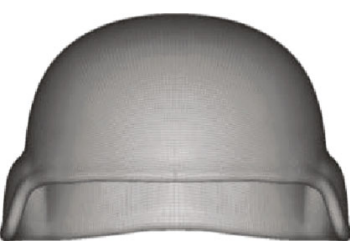

(a) Helmet casing

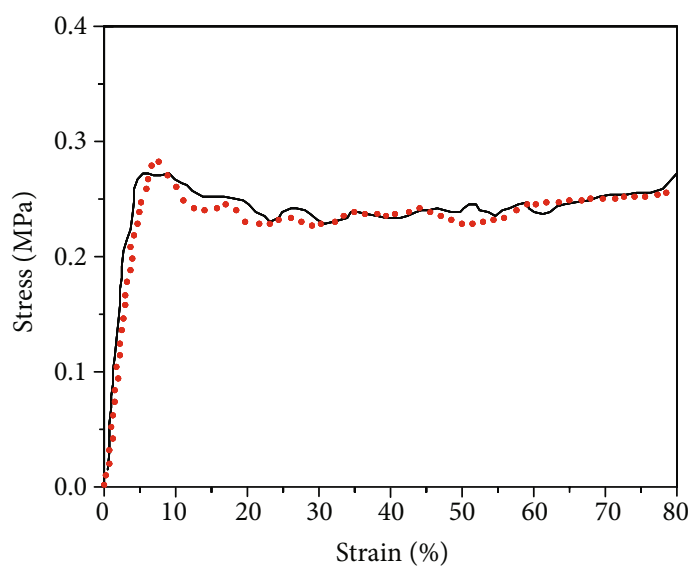

Experiment curve

.... Fitting curve

(c) Foam stress-strain curve
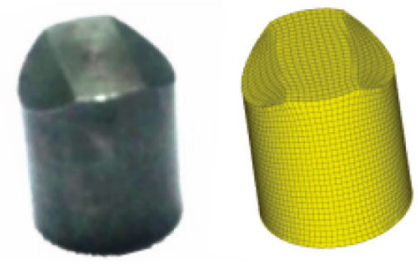

Fragmentation bullet

(d) Bullet models

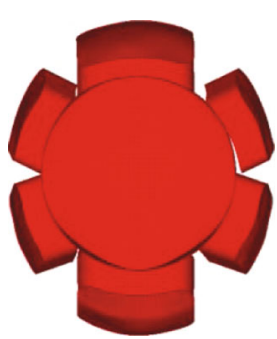

(b) Helmet built-in foam

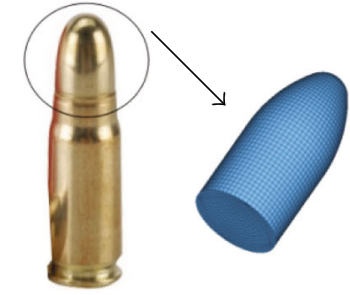

$9 \mathrm{~mm}$ handgun bullet

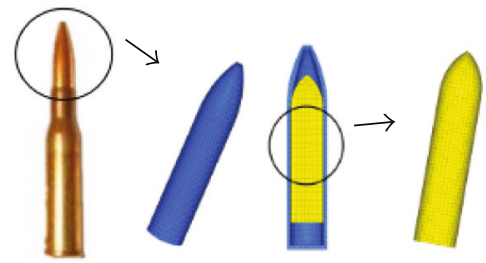

$7.62 \mathrm{~mm}$ rifle bullet

Figure 1: Bullet-proof helmet model and bullet model.

TABLE 1: Bullet material parameters.

\begin{tabular}{lccccc}
\hline Projectile type & $\rho\left(\mathrm{kg} / \mathrm{m}^{3}\right)$ & $E(\mathrm{GPa})$ & $v$ & Yield stress $(\mathrm{GPa})$ & Tangent modulus $(\mathrm{GPa})$ \\
\hline Handgun bullet [20] & 8110 & 210 & 0.3 & 0.792 & 21 \\
Fragmentation bullet [23] & 7830 & 206.8 & 0.3 & & \\
Rifle bullet armor [24] & 8960 & 124 & 0.3 & & \\
Rifle bullet steel core [24] & 7850 & 206 & 0.3 & & \\
\hline
\end{tabular}

2.2. Verification of Finite Element Models. The brain pressures and skull responses of the brain finite element model have been verified based on the relevant literature of Cai et al. [25]. This study involved brain displacements, so the brain-skull relative displacements were verified by the brain finite element model with reference to the Hardy et al. experiment [27].

The C383-T3 high-intensity frontal impact test was used to evaluate the relative displacement of the brain. Invert the head model and select the nodes which are close to the position of the NDT identifier in the Hardy et al. experiment. The nodes a1-a6 are the identification of the anterior humerus to the parietal region and p1-p6 are the posterior occiput to the parietal region; the distance of each marker is approximately $10 \mathrm{~mm}$. The head center of gravity which is automatically calculated by HyperMesh and the node positions are shown in Figure 2; the relative displacement of each node and the skull is calculated. Figure 3 shows the translational acceleration 


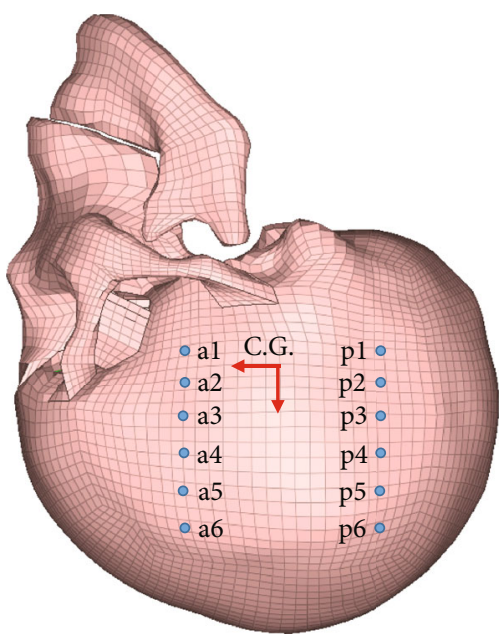

FIgURE 2: Schematic diagram of head centroid and nodes.

and rotational acceleration measured by the Hardy et al. experiment at the head center of gravity and used as loading conditions on the skull which was modeled as rigid.

The $80 \mathrm{~ms}$ head motion response process is simulated, and the relative displacement trajectories of the brain are shown in Figure 4. Overall, the selected nodes in the simulation are basically ring-shaped with respect to the movement of the skull during the impact process, which is consistent with the experimentally obtained motion trajectory.

The brain-skull relative displacements of the simulation and experiment are shown in Figure 5. It can be seen from the figure that the relative displacements of the nodes in the $X$ and $Z$ directions are basically consistent with the experimental results, and the peak is slightly lower than the experimental results. The difference in results may be due to differences in geometric and material properties between the experimental and finite element models, as well as the deviation in the location of experimental test points.

2.3. Research Methods of BHBT. Because the foam material is soft, after wearing a bullet-proof helmet, the brain will have a certain compression deformation on the initial soft foam. In the simulation, the established foam finite element model is prestressed by HyperMesh in order to simulate the response of the bullet-proof helmet in the real environment. The prestressing process is to fix the head and apply gravity on the helmet; the outside of the foam will be deformed to fit the helmet and the inside of the foam will be deformed to fit the head [28]. The final constructed finite element models with detailed anatomical structure are shown in Figures 6(a) and 6(b); the assembly of the bullet- proof helmet, the human brain, and the foam is shown in Figure 6(c). We define the contact between the helmet and the foam and the foam and the scalp as face-to-face contact.

We use the established and verified human brain biomechanical model and the bullet-proof helmet finite element model to study the parameters of brain injury with different impact directions, speeds and structures. We test the location shown in Figure 7 to obtain the amount of deformation on the back of the helmet, the skull stress (at the skull), the abso- lute displacement of the brain (at the cerebrum), and the intracranial pressure (at the dura mater) and evaluate the damage, corresponding to the test point locations which are shown in Table 2. Since the impact time is extremely short, the fixed problem of the head model is not considered. Firstly, the helmet-cranial model was impacted on the frontal, rear, top, left side, and right side of the bullet-proof helmet at a speed of $400 \mathrm{~m} / \mathrm{s}$ by the opponent's bullets to study the damage of the brain in different impact directions. Then, the helmet-cranial model was impacted by bullets at $400 \mathrm{~m} / \mathrm{s}$, $420 \mathrm{~m} / \mathrm{s}, 440 \mathrm{~m} / \mathrm{s}$, and $460 \mathrm{~m} / \mathrm{s}$ from the right. Also, the helmet-cranial model was impacted by a handgun bullet, fragmentation bullet, and rifle bullet with the same kinetic energy of $360 \mathrm{~J}$ on the front of the helmet to simulate the impact of different structures on the brain injury.

\section{Result}

3.1. Influence of Different Impact Directions on Helmet Deformation. The simulation results of the handgun bullet impacting the bullet-proof helmet from different impact directions at $400 \mathrm{~m} / \mathrm{s}$ are shown in Figure 8 . The damage shape of the helmet is circular when impacted in different directions. As shown in Figure 8(a), the BFD value of the output point A (output point position as shown in Figure 7) is different at different positions, and the BFD of the front impact has the maximum value $(12.63 \mathrm{~mm}$ at $0.09 \mathrm{~ms})$, and for the top collision $(10.35 \mathrm{~mm}$ at $0.07 \mathrm{~ms})$, backward collision $(9.021 \mathrm{~mm}$ at $0.11 \mathrm{~ms})$, right impact $(11.46 \mathrm{~mm}$ at $0.14 \mathrm{~ms})$, and left impact $(11.38 \mathrm{~mm}$ at $0.1 \mathrm{~ms})$, the result is basically consistent with the experiments done by Rodríguez-Millán et al. [14]. The trend of the BFD value of different impact directions with time is basically the same. As shown in Figure 8(b), in the change of the model displacement with the time in frontal impact, the bullet contacts the helmet at $t=0.01 \mathrm{~ms}$, the helmet begins to deform, and the skull begins to deform at $t=0.07 \mathrm{~ms}$, The BFD maximum reach at $t=0.09 \mathrm{~ms}$.

3.2. Influence of Different Bullet Speeds on Brain Injury. The simulation results of different bullet speeds impacting the right direction of bullet-proof helmets are shown in Figure 9. The stress cloud map and brain displacements of the cranial skull caused by different bullet speeds impacting bullet-proof helmets are shown in Figure 9. The higher the speed and the greater the BFD value, brain displacement, skull stress, and dura mater pressure, the more serious is the damage of the brain.

3.3. Influence of Different Bullet Structures on Brain Injury under the Same Kinetic Energy. Under the same $360 \mathrm{~J}$ kinetic energy, the speed of the fragmentation is $798 \mathrm{~m} / \mathrm{s}$, the speed of the $9 \mathrm{~mm}$ handgun bullet is $300 \mathrm{~m} / \mathrm{s}$, and the speed of the $7.62 \mathrm{~mm}$ rifle is $256 \mathrm{~m} / \mathrm{s}$. The simulation results of different bullet structures impacting bullet-proof helmets are shown in Figure 10. The dura mater pressure maps of different bullet structures impacting the bullet-proof helmet are shown in Figure $10(\mathrm{a})$. The highest dura mater pressure is $245.1 \mathrm{kPa}$ for the $9 \mathrm{~mm}$ handgun bullet, $238.2 \mathrm{kPa}$ for the rifle bullet, 

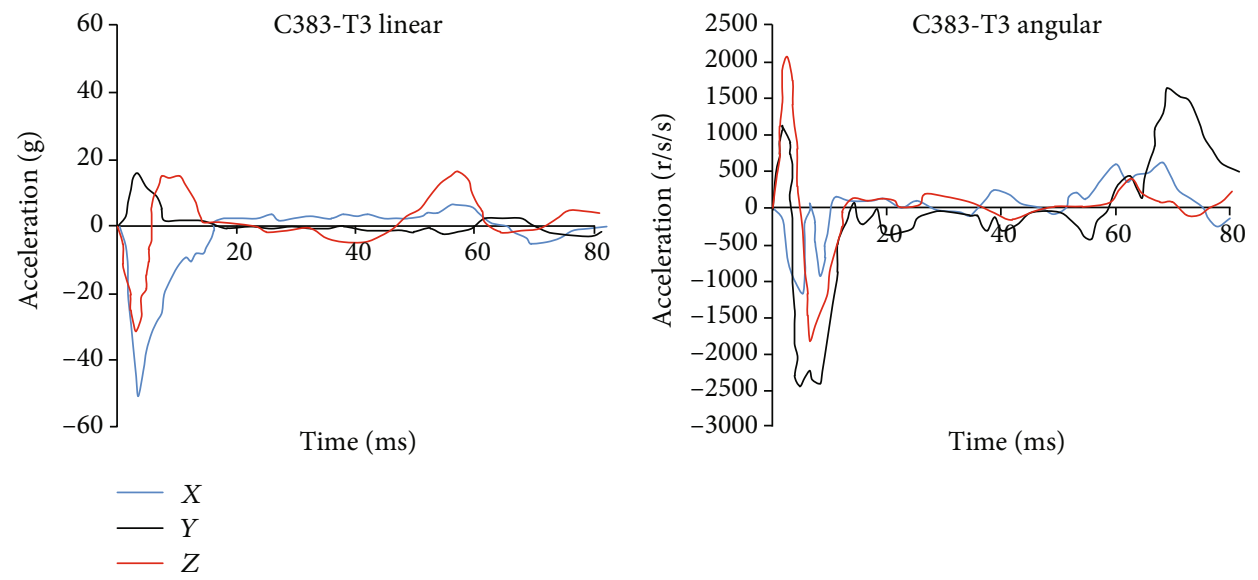

FIgURE 3: The linear and angular accelerations of C383-T3 [27].

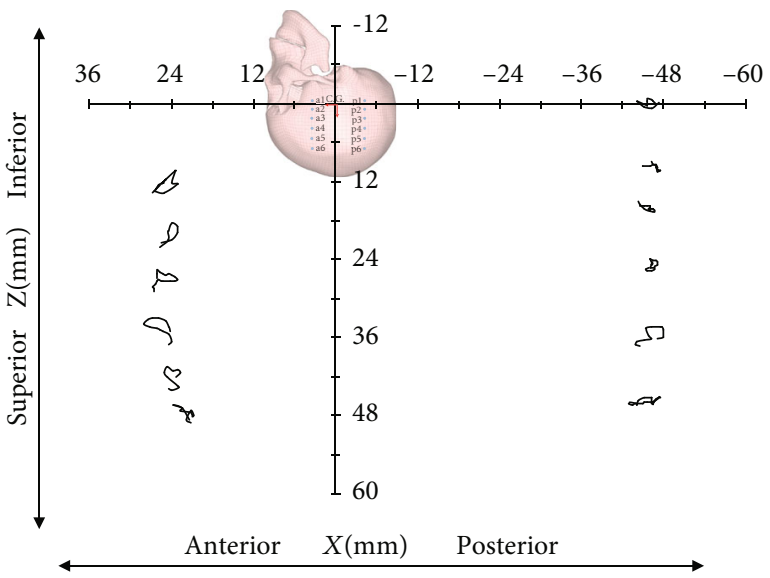

FIGURE 4: Simulation of brain skull relative displacements.

and $225.4 \mathrm{kPa}$ for the fragmentation bullet. Three kinds of bullets impacting bullet-proof helmets to obtain the dura mater pressure output point $\mathrm{D}_{1}$ (output point position as shown in Figure 7) are shown in Figure 10(b). The small mass fragment has the first dura mater pressure change, and the last dura mater pressure change is caused by the largest mass rifle. The BFD values of the output point $A_{1}$ under the three types of bullets impacting bullet-proof helmets are shown in Figure $10(\mathrm{c})$. When the maximum deformation is reached, the fragmentation bullet is the fastest, the handgun bullet is the second, and the rifle bullet is the slowest. The maximum deformation degree of the helmet is the largest for the fragmentation bullet with $7.1 \mathrm{~mm}$, followed by the rifle bullet with $6.8 \mathrm{~mm}$, and the lowest peak of the handgun bullet is $5.6 \mathrm{~mm}$.

\section{Discussion}

The core question of the BHBT and protective performance of bullet-proof helmets is whether it can propose a protective structure and protection method based on real human brain injury, instead of the parameters and methods based on the currently used dummy brain, sludge, or gelatin. At present, the V50 method [16] can accurately evaluate the penetration resistance of the helmet, but it cannot effectively evaluate the BHBT. Therefore, the focus of research and the primary task are to evaluate the index and criteria of BHBT and then further guide the design of the helmet through a large number of experiments, parameter research, and optimization methods.

In this paper, the high-precision human brain biomechanical model is established. Under the LS-DYNA environment, the BHBT caused by the bullet impacting the helmet with high speed is simulated and verified, and the analysis and parameters are studied. The skull fracture was evaluated by the stress of the skull, and the brain injury was evaluated by brain displacement and dura mater pressure. The simulation results of the brain model under high-speed impact are consistent with the overall trend of Raymond et al. [29]. The results show that the established brain biomechanical model can correctly reflect the biomechanical response of the human brain; it has good sensitivity to brain dynamic response under different loading conditions and different parameters, which can provide reference for the evaluation of BHBT and the optimization design of subsequent helmets.

It can be seen from the parametric study that there is a significant difference in the damage of the bullet from the bullet-proof helmet in different directions. Studies have shown that BHBT is related to BFD [16]. The BFD of frontal, rear, top, right, and left obtained from different impact directions in this paper are basically consistent with Rodríguez-Millán et al. [14] and others. The comparisons are shown in Table 3 . With regard to the trend of the BFD value with time in different impact directions and the change of the model displacement with time in the front, $\mathrm{Li}$ et al. [16], Rodríguez-Millán et al. [14], and others also reported similar behavior. The reasons for the differences may be differences in the simulation model, differences in geometric and material properties between different samples, and deviation of experimental test points. The human brain biomechanical model used for this paper includes the scalp, hard bone tissue, brain tissue, and soft tissue, which is closer to the real situation of the human brain. The simulation results 

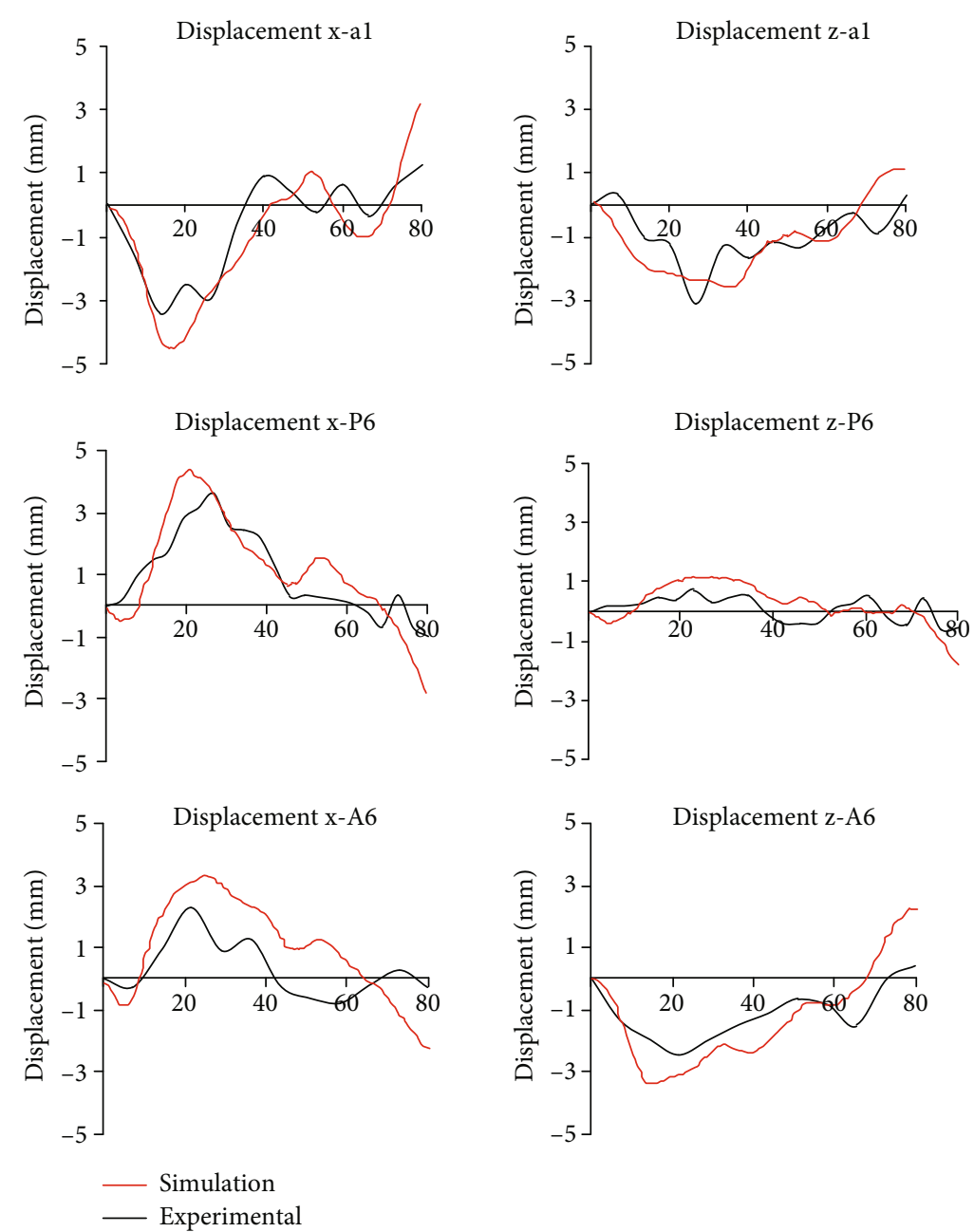

FIGURE 5: Comparison of relative displacement curves of brain in C383-T3 experiment and simulation.

further prove the accuracy of the model and accurately reflect the biomechanical response of BHBT caused by the bullet impacting the helmet.

The peaks of BHBT from different directions are shown in Figure 11. The brain injury is related to the curvature of the helmet. The larger the deformation of the helmet is, the smaller the brain injury is. The BFD value on the right side is a little larger than that on the left side; the skull stress, brain displacement, and dura mater pressure peaks are smaller than the left. The damage to the brain is also related to the layout of the helmet foam interior. The foam padding area on the left and right sides which consists of two foams with gaps is smaller than that on the other three directions. The simulation results show that the brain displacement and the skull stress are about twice higher when impacted by the left and right sides than when impacted by the front, back, and top.

The injury caused by a high-speed bullet impacting the helmet becomes more serious with the increase of the bullet speed. As the speed increases, the stress of the skull increases and the brain displacement occurs. Rafaels et al. [3] reported that there was no fracture in the low-speed lower brain, a crack at the medium speed, and a fracture at high speed, and there were brain displacements in the seven brain tests. The results of this paper are consistent with the experiment mentioned above.

The peaks of BHBT caused by different bullet velocities impacting bullet-proof helmets are shown in Figure 12. BFD value, skull stress, brain displacement, and peak dura mater pressure can better reflect the brain injury. Simulations show that BFD, skull stress, brain displacement, and dura mater pressure peaks increase with speed.

There are differences in the degree of brain injury caused by different bullet structures impacting bullet-proof helmets under the same kinetic energy. This paper refers to Wang et al. [30] and other people's research on the damage characteristics of landrace which wear on them the body armor with impacts of different structures of rifle bullets. The initial energy of the bullets of three different types of missile structures was adjusted to the same by changing the speed of the bullets. In the same situation as the kinetic energy studied in the literature, different structures are consistent with different brain damage results.

The peaks of the BHBT caused by different bullet structures impacting bullet-proof helmets are shown in Figure 13. When the different structures are impacted on the bullet- 


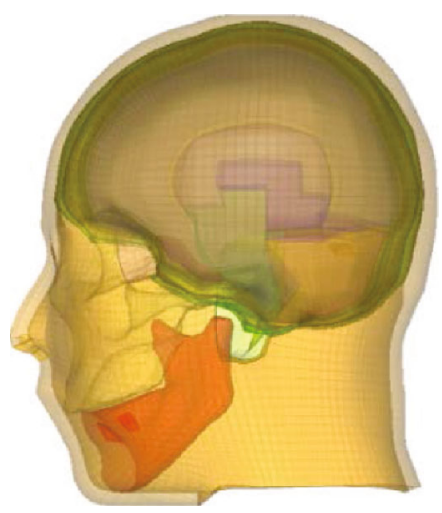

(a)

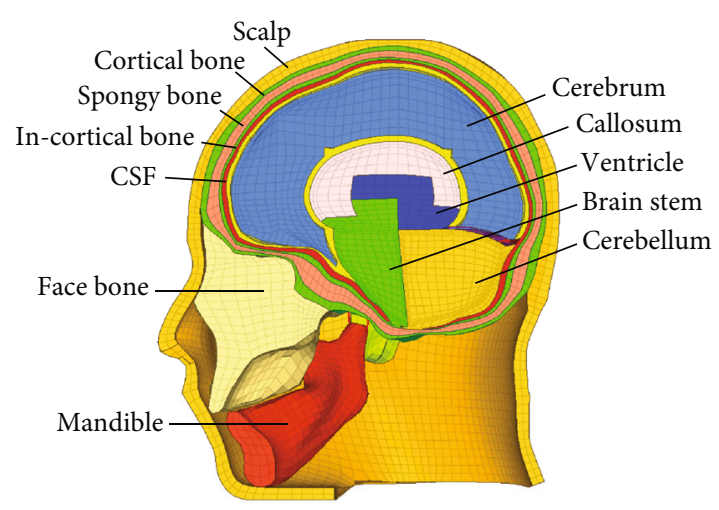

(b)

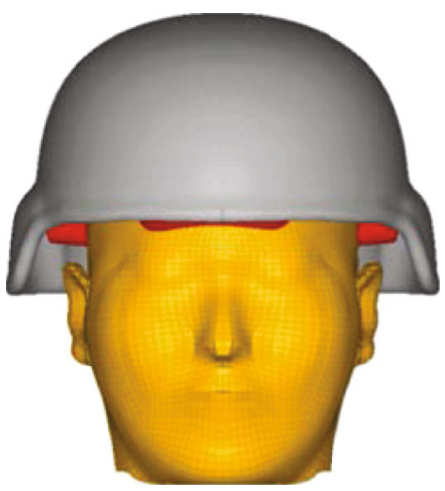

(c)

Figure 6: Finite element models.
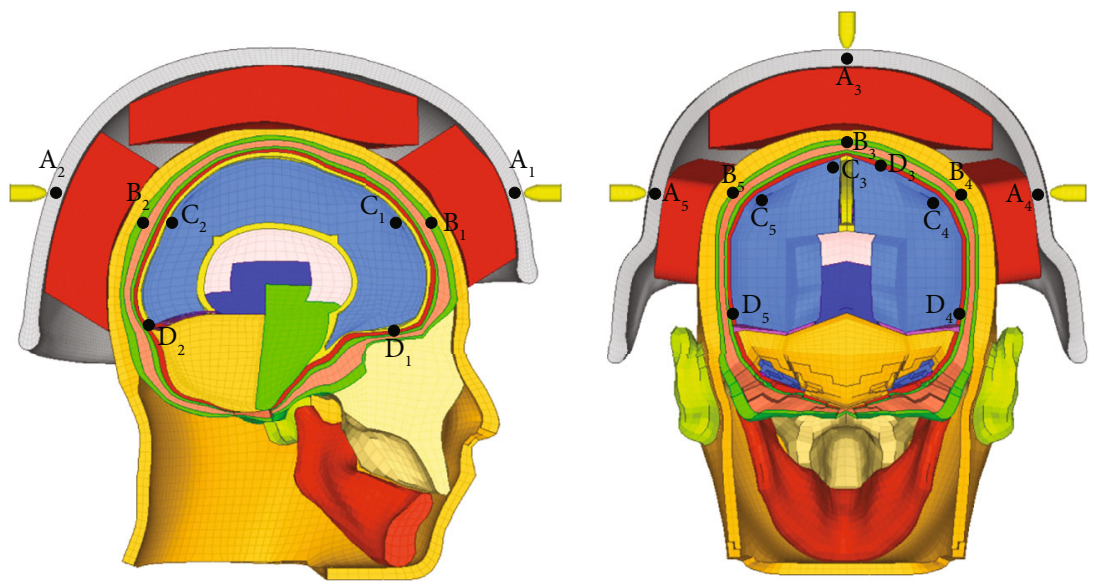

Figure 7: Measurement location of different impact points.

TABLE 2: Corresponding serial number of measurement impact point.

\begin{tabular}{lccccc}
\hline & Frontal & Rear & Top & Right & Left \\
\hline BFD & $\mathrm{A}_{1}$ & $\mathrm{~A}_{2}$ & $\mathrm{~A}_{3}$ & $\mathrm{~A}_{4}$ & $\mathrm{~A}_{5}$ \\
Skull stress & $\mathrm{B}_{1}$ & $\mathrm{~B}_{2}$ & $\mathrm{~B}_{3}$ & $\mathrm{~B}_{4}$ & $\mathrm{~B}_{5}$ \\
Brain displacement & $\mathrm{C}_{1}$ & $\mathrm{C}_{2}$ & $\mathrm{C}_{3}$ & $\mathrm{C}_{4}$ & $\mathrm{C}_{5}$ \\
Intracranial pressure & $\mathrm{D}_{1}$ & $\mathrm{D}_{2}$ & $\mathrm{D}_{3}$ & $\mathrm{D}_{4}$ & $\mathrm{D}_{5}$ \\
\hline
\end{tabular}

proof helmet, the energy release mode is different due to the structure of the missile body and the high velocity collision between the projectile and the bullet-proof material; the damage caused to different parts of the brain is inconsistent. The velocity of the fragmentation bullet is the largest, which results in the largest BFD value and the largest brain displacement peak, but the skull stress peak is the smallest. The $9 \mathrm{~mm}$ handgun bullet is smoother than the $7.62 \mathrm{~mm}$ rifle bullet, which results in the smallest BFD value, the lowest brain displacement, but the largest skull stress peak. 

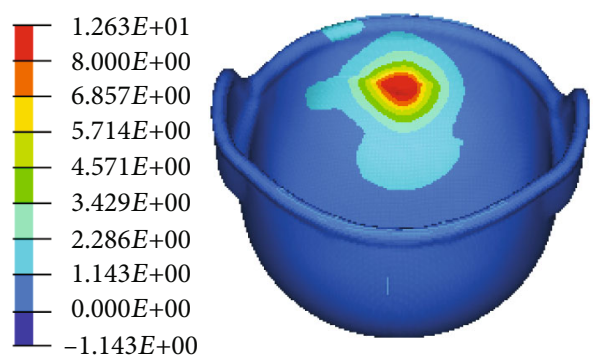

Fontal
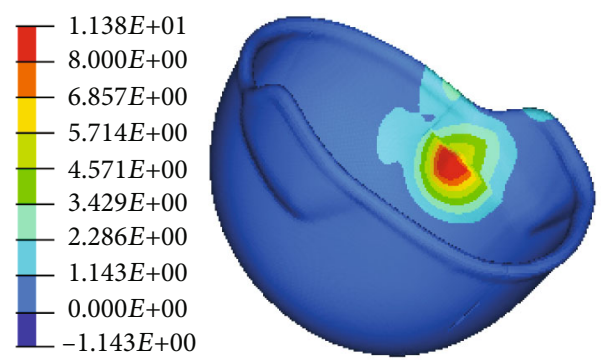

Left

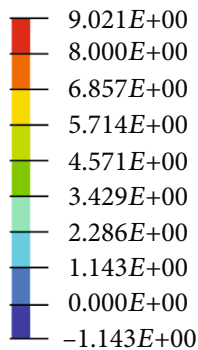

$-1.143 E+00$

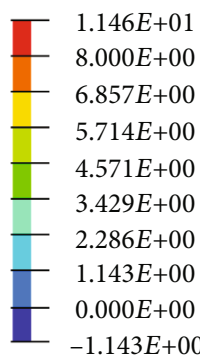

$-1.143 E+00$

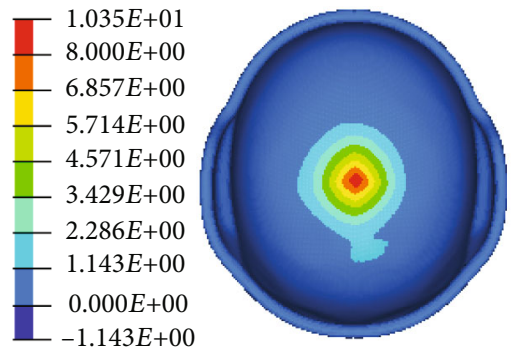

Top
Right

Rear

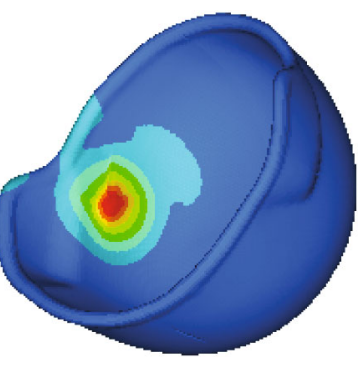

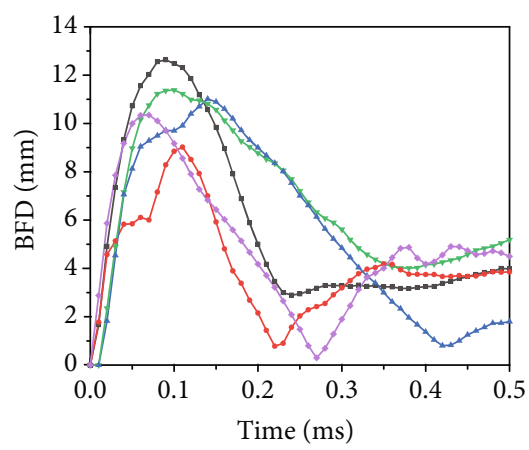

(a) BFD (mm)

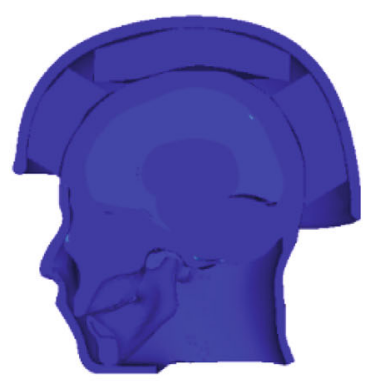

$0 \mathrm{~ms}$

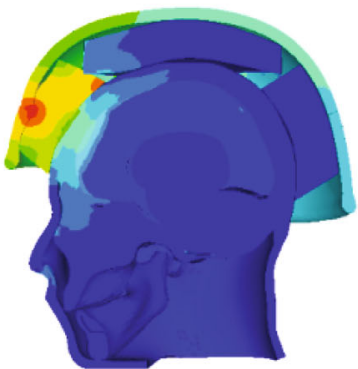

$0.14 \mathrm{~ms}$

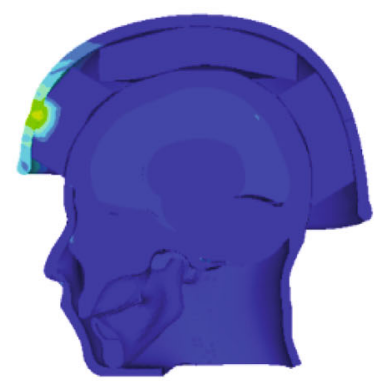

$0.01 \mathrm{~ms}$

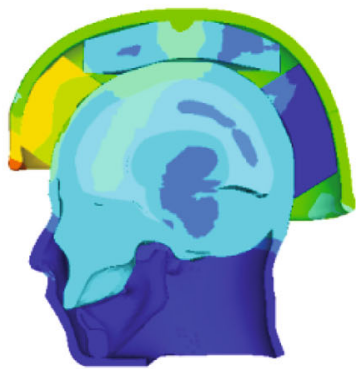

$0.35 \mathrm{~ms}$

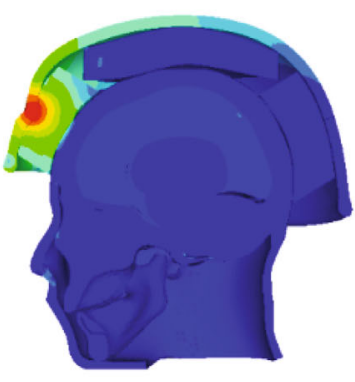

$0.07 \mathrm{~ms}$

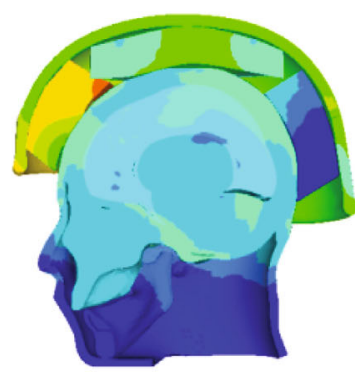

$0.5 \mathrm{~ms}$

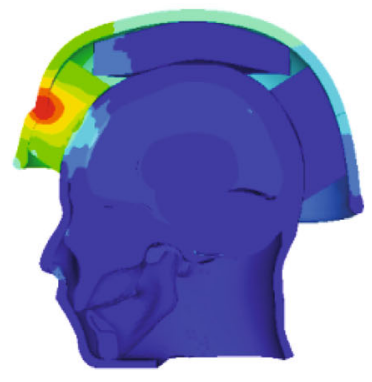

$0.1 \mathrm{~ms}$

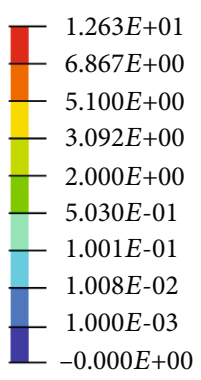

Displacement

(b) Helmet and brain deformation during frontal impact

Figure 8: Helmet and brain deformation.

\section{Conclusion}

The results show that the brain model used in this paper can reflect the biomechanical response of the human brain and has sensitivity to brain dynamic response under different impact conditions. The impact position, impact velocity, and structure of the bullet have a significant influence on the skull and brain responses. In particular, the frontal impact of the skull produces the largest amount of BFD, and when the impact directions are from the side, the skull 


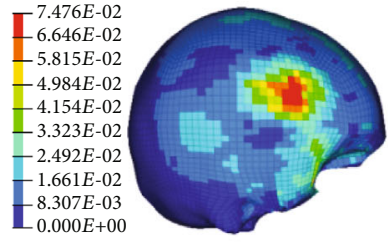

$400 \mathrm{~m} / \mathrm{s}$

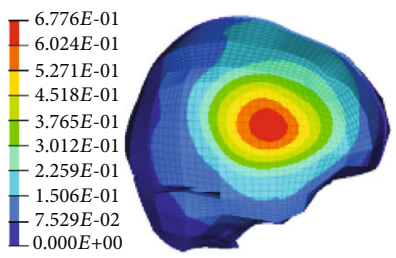

$400 \mathrm{~m} / \mathrm{s}$

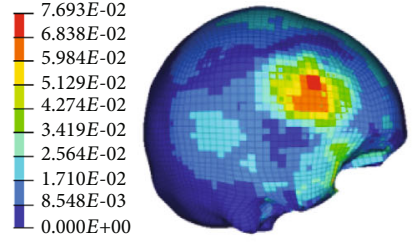

$420 \mathrm{~m} / \mathrm{s}$

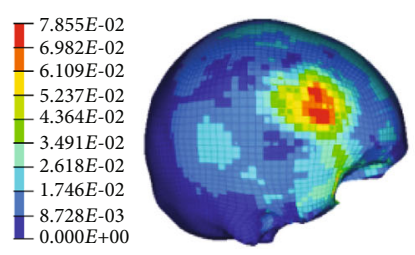

$440 \mathrm{~m} / \mathrm{s}$

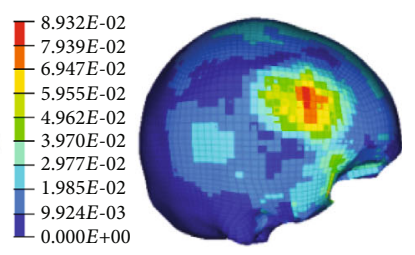

$460 \mathrm{~m} / \mathrm{s}$

(a) Skull stress at different bullet speeds (GPa)
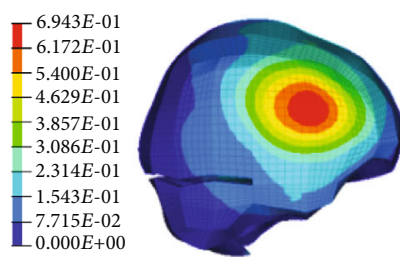

$420 \mathrm{~m} / \mathrm{s}$

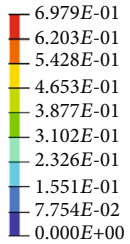

$-7.754 E-02$
$-0.000 E+00$

$440 \mathrm{~m} / \mathrm{s}$
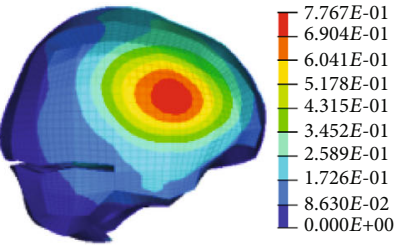

$-8.630 E-02$
$0.000 E+00$
$460 \mathrm{~m} / \mathrm{s}$

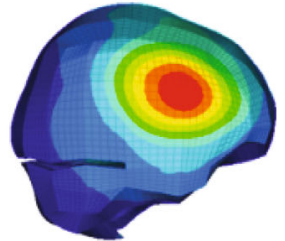

(b) Brain displacement at different bullet speeds $(\mathrm{mm})$

Figure 9: Head response at different bullet speeds.

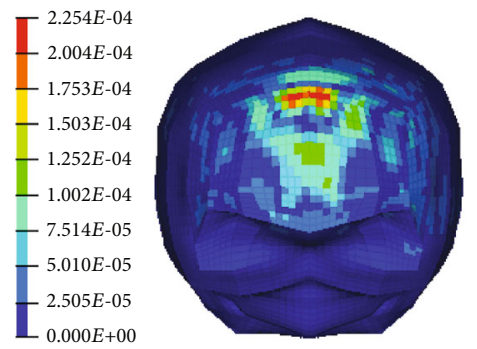

Fragmentation bullet

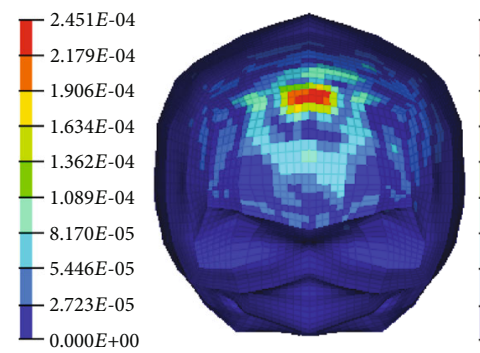

$9 \mathrm{~mm}$ handgun bullet
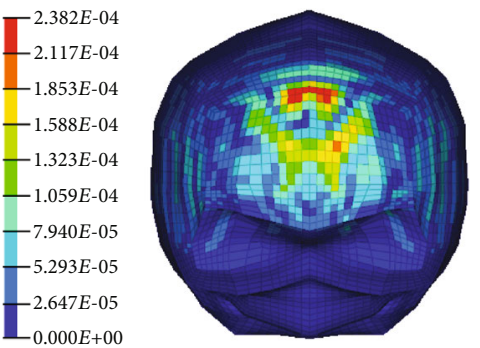

$7.62 \mathrm{~mm}$ rifle bullet

(a) Dura mater pressure under different bullet structures (Gpa)

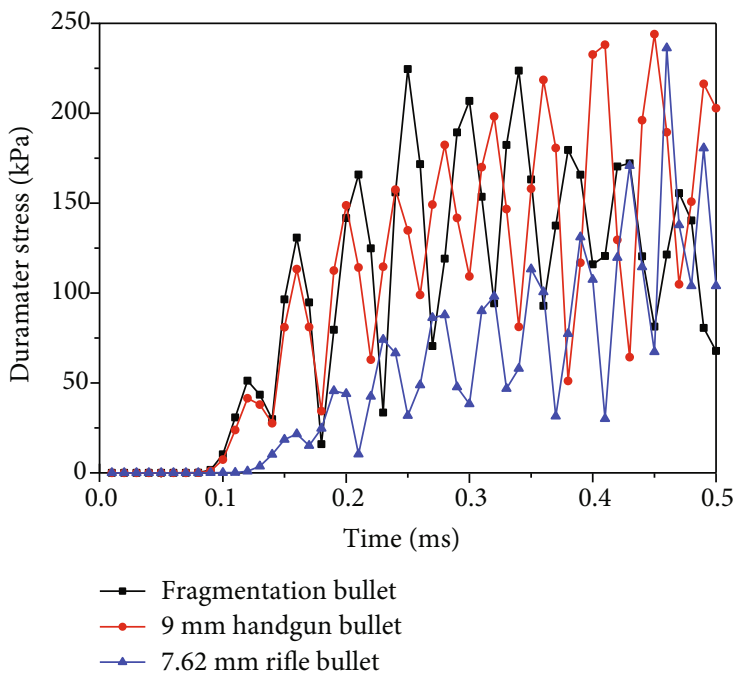

(b) Dura mater pressure under different bullet structures

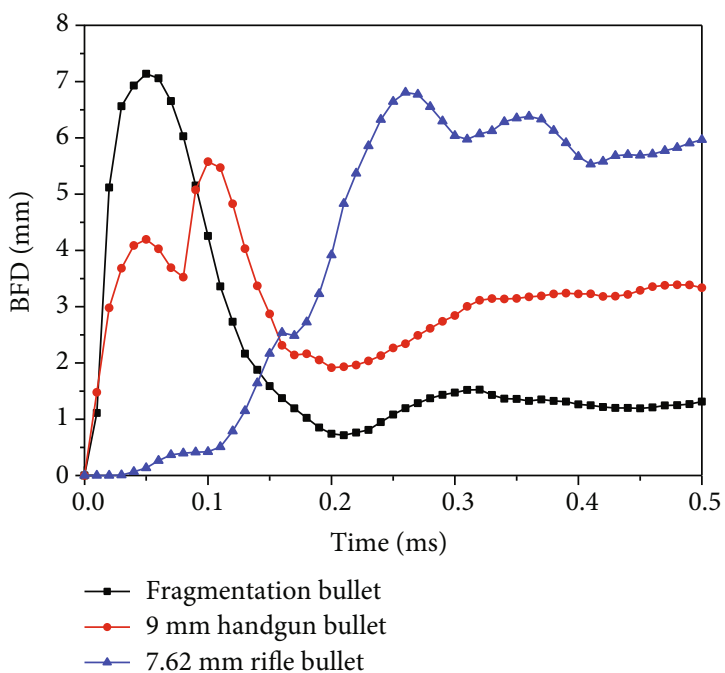

(c) BFD under different bullet structures

Figure 10: Brain response under different bullet structures.

stress is about twice higher than from other directions; as the impact velocity increases, the BFD, brain displacement, skull stress, and dura mater pressure increase, and the brain damage caused by different structural bullet bodies is different under the condition of the same kinetic energy and the skull stress caused by the handgun bullet is the largest. The findings indicate that when a bullet impacts on the bullet-proof helmet, it has a higher probability of causing 
TABLE 3: BFD values of different impact directions compared with the literature.

\begin{tabular}{|c|c|c|c|c|c|}
\hline & Frontal (mm) & Rear $(\mathrm{mm})$ & Top $(\mathrm{mm})$ & Right (mm) & Left $(\mathrm{mm})$ \\
\hline Simulation results & 12.63 & 9.021 & 10.35 & 11.46 & 11.38 \\
\hline Experiment in literature [14] & 12 & 9 & 11 & 11 & 6 \\
\hline
\end{tabular}
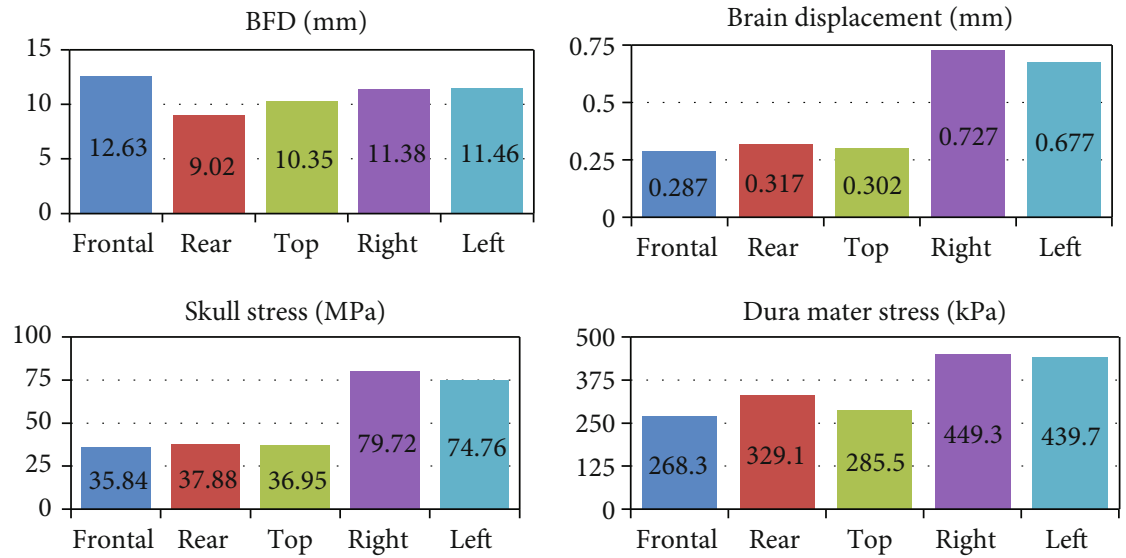

FIgURE 11: Comparison of peak brain damage in different directions.
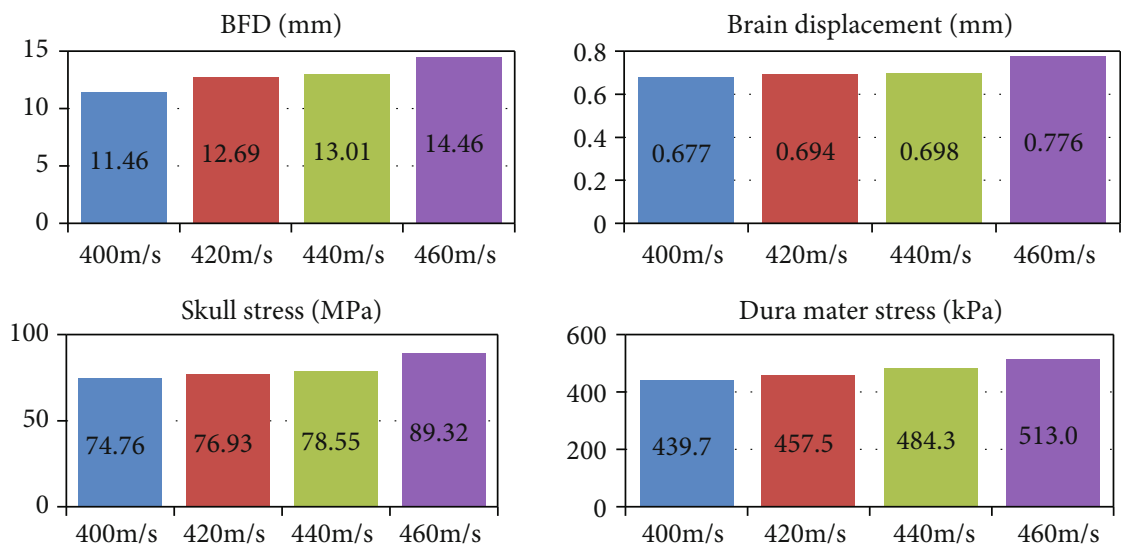

FIGURE 12: Comparison of peak values impacted by different projectiles.
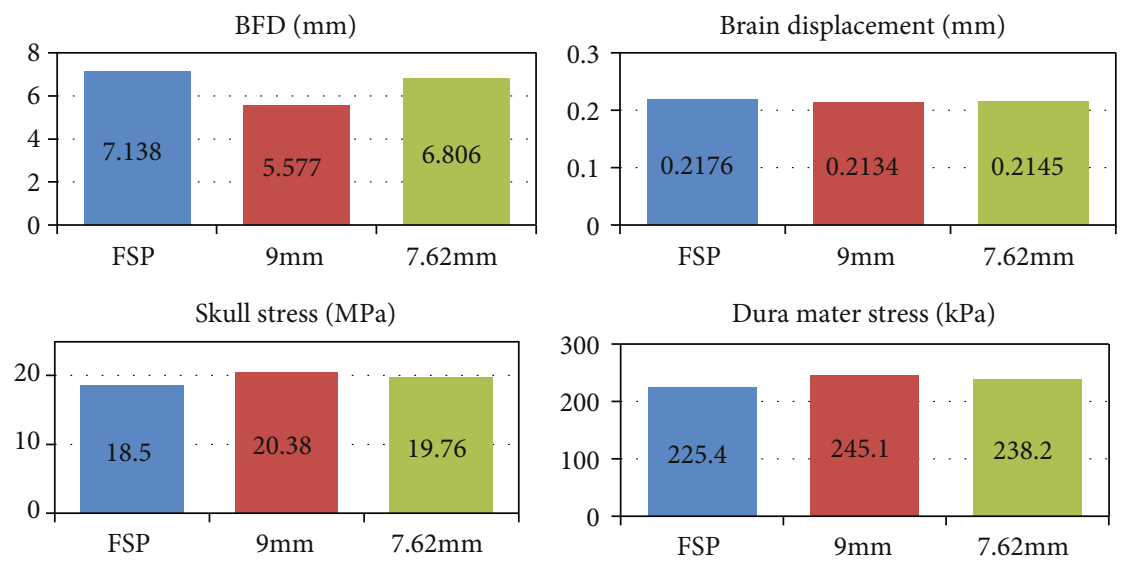

Figure 13: Comparison of peak values impacted by different projectile structures. 
brain displacement and intracranial high pressure. Intracranial pressure higher than $235 \mathrm{kPa}$ could result in serious brain injury, and the tensile fracture stress for the skull is around $75 \mathrm{MPa}$ [28]. The brain damage caused by different structural bodies is different when the kinetic energy is consistent. Although we have studied the brain injury caused by different bullet speeds, different impact directions, and different bullet structures, there are still many shortcomings to be further studied, such as the impact angle. As mentioned in the article [16], a $45 \mathrm{deg}$ oblique frontal impact leads to a lower head injury risk than a $90 \mathrm{deg}$ frontal impact. In the future, we will continue to study the damage of the brain from different impact angles and so on. This paper can provide reference value for helmet optimization design and antielasticity evaluation and provide the theoretical basis for protection and rescue.

\section{Data Availability}

The data used to support the findings of this study are available from the corresponding author upon request.

\section{Conflicts of Interest}

The authors declare that there is no conflict of interest regarding the publication of this paper.

\section{Acknowledgments}

We acknowledge the support from the National Natural Science Foundation of China (Grant Nos. 11972158, 81973871, and 51805162) and the Educational Commission of Hunan Province of China (Grant Nos. 17A068 and 18A188).

\section{References}

[1] L. Cannon, "Behind armour blunt trauma-an emerging problem," Journal of the Royal Army Medical Corps, vol. 147, no. 1, pp. 87-96, 2001.

[2] C. J. Freitas, J. T. Mathis, N. Scott, R. P. Bigger, and J. Mackiewicz, "Dynamic response due to behind helmet blunt trauma measured with a human head surrogate," International Journal of Medical Sciences, vol. 11, no. 5, pp. 409-425, 2014.

[3] K. A. Rafaels, H. C. Cutcliffe, R. S. Salzar et al., "Injuries of the head from backface deformation of ballistic protective helmets under ballistic impact," Journal of Forensic Sciences, vol. 60, no. 1, pp. 219-225, 2015.

[4] M. S. Jazi, A. Rezaei, G. Karami, F. Azarmi, and M. Ziejewski, "A computational study of influence of helmet padding materials on the human brain under ballistic impacts," Computer Methods in Biomechanics and Biomedical Engineering, vol. 17, no. 12, pp. 1368-1382, 2012.

[5] J. Yang and J. Dai, "Simulation-based assessment of rear effect to ballistic helmet impact," Computer-Aided Design and Applications, vol. 7, no. 1, pp. 59-73, 2010.

[6] F. A. Pintar, M. M. G. M. Philippens, J. Y. Zhang, and N. Yoganandan, "Methodology to determine skull bone and brain responses from ballistic helmet- to-head contact loading using experiments and finite element analysis," Medical Engineering \& Physics, vol. 35, no. 11, pp. 1682-1687, 2013.
[7] K. M. Tse, L. B. Tan, S. J. Lee, S. P. Lim, and H. P. Lee, "Development and validation of two subject-specific finite element models of human head against three cadaveric experiments," International Journal for Numerical Methods in Biomedical Engineering, vol. 30, no. 3, pp. 397-415, 2014.

[8] H.-S. Kang, R. Willinger, B. M. Diaw, and B. Chinn, "Validation of a $3 \mathrm{D}$ anatomic human head model and replication of head impact in motorcycle accident by finite element modeling," SAE Transactions, vol. 106, pp. 38493858, 1997.

[9] J. S. Ruan, T. B. Khalil, and A. I. King, "Finite element modeling of direct head impact," SAE Technical Paper, 1993.

[10] S. Kleiven, "Predictors for traumatic brain injuries evaluated through accident reconstructions," Stapp Car Crash Journal, vol. 51, pp. 81-114, 2007.

[11] T. J. Horgan and M. D. Gilchrist, "The creation of threedimensional finite element models for simulating head impact biomechanics," International Journal of Crashworthiness, vol. 8, no. 4, pp. 353-366, 2003.

[12] H. Mao, L. Zhang, B. Jiang et al., "Development of a finite element human head model partially validated with thirty five experimental cases," Journal of Biomechanical Engineering, vol. 135, no. 11, pp. 111002-111015, 2013.

[13] R. T. Cotton, C. W. Pearce, P. G. Young et al., "Development of a geometrically accurate and adaptable finite element head model for impact simulation: the Naval Research Laboratory-Simpleware Head Model," Computer Methods in Biomechanics and Biomedical Engineering, vol. 19, no. 1, pp. 101-113, 2016.

[14] M. Rodríguez-Millán, T. Ito, J. A. Loya, A. Olmedo, and M. H. Miguélez, "Development of numerical model for ballistic resistance evaluation of combat helmet and experimental validation," Materials \& Design, vol. 110, pp. 391-403, 2016.

[15] E. Palta, H. Fang, and D. C. Weggel, "Finite element analysis of the Advanced Combat Helmet under various ballistic impacts," International Journal of Impact Engineering, vol. 112, pp. 125-143, 2018.

[16] X. G. Li, X. L. Gao, and S. Kleiven, "Behind helmet blunt trauma induced by ballistic impact: a computational model," International Journal of Impact Engineering, vol. 91, pp. 5667, 2016.

[17] C. Y. Tham, V. B. C. Tan, and H. P. Lee, "Ballistic impact of a Kevlar $^{\circledR}$ helmet: experiment and simulations," International Journal of Impact Engineering, vol. 35, no. 5, pp. 304-318, 2008.

[18] H. Liu, J. Kang, J. Chen, G. Li, X. Li, and J. Wang, "Intracranial pressure response to non-penetrating ballistic impact: an experimental study using a pig physical head model and live pigs," International Journal of Medical Sciences, vol. 9, no. 8, pp. 655-664, 2012.

[19] M. Palomar, E. Lozano-Mínguez, M. Rodríguez-Millán, M. H. Miguélez, and E. Giner, "Relevant factors in the design of composite ballistic helmets," Composite Structures, vol. 201, pp. 49-61, 2018.

[20] L. B. Tan, K. M. Tse, H. P. Lee, V. B. C. Tan, and S. P. Lim, "Performance of an advanced combat helmet with different interior cushioning systems in ballistic impact: experiments and finite element simulations," International Journal of Impact Engineering, vol. 50, pp. 99-112, 2012.

[21] M. Aare and S. Kleiven, "Evaluation of head response to ballistic helmet impacts using the finite element method," 
International Journal of Impact Engineering, vol. 34, no. 3, pp. 596-608, 2007.

[22] Z. Cai, Z. Li, J. Dong, Z. Mao, L. Wang, and C. J. Xian, “A study on protective performance of bullet-proof helmet under impact loading," Journal of Vibroengineering, vol. 18, no. 4, pp. 2495-2507, 2016.

[23] J. van Hoof, D. S. Cronin, M. J. Worswick, K. V. Williams, and D. Nandlall, "Numerical head and composite helmet models to predict blunt trauma," in 19th International Symposium of Ballistics, pp. 921-928, Interlaken, Switzerland, 2001..

[24] N. KılıÇ, B. Ekici, and S. Hartomacıoğlu, "Determination of penetration depth at high velocity impact using finite element method and artificial neural network tools," Defence Technology, vol. 11, no. 2, pp. 110-122, 2015.

[25] Z. Cai, Y. Xia, Z. Bao, and H. Mao, "Creating a human head finite element model using a multi-block approach for predicting skull response and brain pressure," Computer Methods in Biomechanics and Biomedical Engineering, vol. 22, no. 2, pp. 169-179, 2019.

[26] Z. Cai, Y. Xia, and X. Huang, “Analyses of pedestrian's headto-windshield impact biomechanical responses and head injuries using a head finite element model," Journal of Mechanics in Medicine and Biology, article 1950063, 2019.

[27] W. N. Hardy, C. D. Foster, M. J. Mason, K. H. Yang, A. I. King, and S. Tashman, "Investigation of head injury mechanisms using neutral density technology and high-speed biplanar X-ray," Stapp Car Crash Journal, vol. 45, p. 337, 2001.

[28] K. M. Tse, L. B. Tan, B. Yang, V. B. Tan, and H. P. Lee, "Effect of helmet liner systems and impact directions on severity of head injuries sustained in ballistic impacts: a finite element (FE) study," Medical \& Biological Engineering \& Computing, vol. 55, no. 4, pp. 641-662, 2017.

[29] D. Raymond, C. van Ee, G. Crawford, and C. Bir, "Tolerance of the skull to blunt ballistic temporo-parietal impact," Journal of Biomechanics, vol. 42, no. 15, pp. 2479-2485, 2009.

[30] L.-q. Wang, X.-n. Lai, B. Zhang, S. Zheng-lin, Y.-f. Huang, and L.-l. Wang, "Characteristics of behind armor blunt trauma produced by bullets with different structural materials: an experimental study," Medical Journal of Chinese Peoples Liberation Army, vol. 38, no. 7, pp. 601-604, 2013. 